\title{
L'école à la recherche du temps perdu
}

Les débats sur l'école au Portugal

\section{Rui Canário}

\section{CpenEdition}

\section{Journals}

Édition électronique

URL : http://journals.openedition.org/ries/3177

DOI : $10.4000 /$ ries. 3177

ISSN : 2261-4265

Éditeur

Centre international d'études pédagogiques

Édition imprimée

Date de publication : 1 septembre 1997

Pagination : 99-113

ISSN : 1254-4590

Référence électronique

Rui Canário, "L'école à la recherche du temps perdu », Revue internationale d'éducation de Sèvres [En ligne], 15 | 1997, mis en ligne le 15 juillet 2013, consulté le 14 novembre 2019. URL : http:// journals.openedition.org/ries/3177 ; DOI : 10.4000/ries.3177

Ce document a été généré automatiquement le 14 novembre 2019.

(c) Tous droits réservés 


\title{
L'école à la recherche du temps perdu
}

\author{
Les débats sur l'école au Portugal
}

Rui Canário

1 L'école est aujourd'hui, au Portugal, un objet qui suscite beaucoup d'attentions, mais aussi des sentiments très divers et très souvent contradictoires. L'école apparaît, d'une part, comme une condition même du progrès et de la modernisation du pays. Le gouvernement actuel (socialiste) a même élu l'école comme un objet de "passion », en transformant cette idée dans un slogan politique. D'autre part, l'école, source de pessimisme et d'insatisfaction peut être perçue comme « inutile » parce qu'elle n'assure que des "emplois sans avenir et mal rémunérés», comme l'affirmait, en titre de première page, le plus influent quotidien du pays ${ }^{1}$.

2 La tension entre ces deux visions opposées de l'institution scolaire constitue non seulement la traduction d'un phénomène plus universel - la crise de légitimité de l'école -, mais elle exprime aussi les particularités d'un système éducatif qui vit encore une phase d'expansion quantitative et qui ressent, simultanément, les conséquences négatives de la «massification ». En fait, une politique de démocratisation de l'accès à l'enseignement n'a commencé vraiment qu'au début des années soixante-dix et on ne peut parler d'un phénomène d'« explosion scolaire » au Portugal qu'après la révolution d'avril 1974.

3 Les besoins de croissance du système scolaire sont encore loin d'être résolus. En effet, selon les données du ministère de l'Éducation (Portugal, 1996), le niveau éducatif de la population (groupe d'âge 15-64 ans) est extrêmement bas : $74 \%$ de cette population a comme niveau maximum six ans de scolarité, tandis que seule une minorité de $5 \%$ possède un niveau supérieur. L'éducation préscolaire n'est assurée qu'à environ la moitié des enfants et un cinquième des jeunes n'arrive pas à terminer la scolarité obligatoire de neuf ans. Cette situation est parallèle avec un débat public très vif sur la «qualité » des apprentissages fournis par l'école, notamment à propos des résultats décevants des examens passés à la fin de l'enseignement secondaire. Des voix s'élèvent 
pour vanter l'école de «jadis », celle qui permettait de "travailler sérieusement » et d'« apprendre vraiment ».

4 De ce point de vue, dans un système scolaire qui allie l'incapacité à faire accomplir la scolarité obligatoire et l'existence d'un «numerus clausus» dans l'accès aux établissements supérieurs publics, on peut dire que l'école se trouve dans la situation (paradoxale) de chercher le temps perdu, dans un sens double :d'une part, parce qu'il lui faut dépasser le «retard» historique dans la construction d'une école de masse ; d'autre part, parce que cette massification engendre la tentation de revenir à un âge d'or du passé, pendant lequel l'école a «bien fonctionné ». En gardant ce cadre comme point de repère, nous essayerons d'identifier quels sont, de notre point de vue, les points critiques du débat actuel sur l'école au Portugal. Notre analyse sera circonscrite au niveau de la scolarité obligatoire.

\section{Réforme ou innovation?}

5 Le premier thème du débat actuel porte sur la méthodologie la plus adéquate à la production de changements délibérés en éducation, en particulier en ce qui concerne les établissements scolaires. En effet, la scène éducative portugaise a été marquée, pendant la dernière décennie, par la mise en place d'une réforme éducative, déclenchée depuis 1986 par l'approbation, au Parlement, d'une loi-cadre. Par ses ambitions, son ampleur et sa méthodologie, cette réforme, qui a repris le modèle dominant pendant les années soixante-dix, s'est traduite par un échec².

Malgré cet échec, il est possible de constater l'existence d'un important potentiel de créativité dans les écoles portugaises, responsables de nombreuses expériences d'innovation bien réussies, dont rend compte la littérature disponible ${ }^{3}$. La possibilité d'élucider ce paradoxe suppose, d'une part, la clarification des limites de la réforme et, d'autre part, la capacité de comprendre que les établissements sont, dans une certaine mesure, condamnés à innover, même pour des raisons de "survivance $»^{4}$. Malgré les réformes, les établissements scolaires changent (et changent les réformes) : que peut nous apprendre cette constatation?

7 Dans le cas portugais, on trouve les ingrédients typiques des réformes classiques, sous la forme de deux stratégies principales. La première consiste à utiliser le pouvoir coercitif de l'État pour imposer («d'en haut») des changements à partir de la production de normes légales. La deuxième stratégie se fonde sur une démarche empirique/rationnelle et se traduit, du point de vue pratique, par un modèle industriel de production de changements qui, d'une façon linéaire, passerait d'une étape de recherche (production d'un prototype) à une étape d'expérimentation (établissements scolaires pilotes) et, finalement, à une étape de généralisation à l'ensemble des établissements.

8 Ces deux stratégies matérialisent une logique instituée de changement, fondée sur une nette séparation - dans l'espace, dans le temps et parmi les acteurs - entre la conception et l'exécution des changements, ce qui engendre deux difficultés majeures : d'une part, le passage du niveau des intentions exprimées dans les lois à celui des actes (limites du changement par décret), d'autre part, le passage du terrain expérimental à la généralisation sur l'ensemble du système éducatif (limites du modèle de recherchedéveloppement). Le processus de réforme ne produit, finalement, que des changements potentiels (des normatifs et des prototypes). Leur transformation d'un stade virtuel à 
un stade réel fait appel à l'émergence d'un processus instituant qui suppose l'invention de nouvelles façons d'agir et d'interagir, de la part des acteurs, dans chaque contexte.

Les études empiriques que nous avons conduites pendant les dernières années, en particulier sur les transformations des bibliothèques scolaires ${ }^{5}$, mettent en évidence des processus de changement, marqués par la complexité et par la singularité (les solutions ont toujours un caractère local bien qu'elles correspondent souvent à des reconfigurations de pratiques qui ont eu leur origine ailleurs). L'établissement scolaire, envisagé comme un " construit social », devient l'unité cruciale où se joue la possibilité de faire naître une dynamique et une logique d'innovation, susceptibles de favoriser le changement non plus du système scolaire, mais plutôt d'un système d'écoles. Trois orientations stratégiques apparaissent comme fondamentales pour fonder une logique de l'innovation.

La première consiste à passer, comme nous le conseille Crozier ${ }^{6}$, d'une culture de solutions à une culture de problèmes. Les problèmes à résoudre sont, tout d'abord, des «constructions sociales", c'est-à-dire qu'ils ne préexistent pas aux situations ni à l'interaction des acteurs; ensuite, ils apparaissent comme indéterminés et ils sont donc réfractaires à la recherche du one best way; finalement, ils ne sont jamais isolés, au contraire, ils s'inscrivent dans le cadre de situations problématiques qui font appel à une approche holistique et contextualisée.

11 La deuxième consiste dans le développement de la capacité d'écoute par rapport aux établissements scolaires. Cette capacité apparaît comme fondamentale pour qu'on puisse mieux connaître ce que font les établissements et favoriser la mise à profit de leur créativité dans l'ensemble du système (mise en place des réseaux).

12 Troisièmement, une rupture avec une vision instrumentale et adaptative du changement devient nécessaire. A une relation avec les établissements basée sur la méfiance, la prescription et le contrôle, on attend de l'Administration un nouveau rôle de régulation, orienté vers l'aide au développement organisationnel des établissements scolaires pour leur permettre d'« apprendre » à bien fonctionner.

13 Ce qui est en jeu, finalement, c'est la possibilité de tirer toutes les conclusions de l'échec de la réforme, en tant que méthodologie ne permettant pas d'aider à construire des réponses pertinentes à la crise de légitimité de l'institution scolaire. Dans le débat qui se poursuit au Portugal depuis quelques années, deux perspectives s'opposent: l'une élabore une démarche déductive (logique de la réforme), l'autre une démarche inductive (logique de l'innovation). Nous avons tendance à penser que la prédominance de la première tend à fonctionner comme un vaccin contre la seconde.

\section{Formation des enseignants}

14 Il n'est pas surprenant que, pendant les dix dernières années, au Portugal, la formation des enseignants (en particulier la formation continue) soit apparue comme une question centrale de la politique éducative. Du point de vue de l'administration, la formation continue, envisagée - selon une logique adaptative - a posteriori aux changements proposés par la réforme, constitue une condition essentielle pour persuader les enseignants et les aider à «bien appliquer» la réforme. Cette attention portée sur la formation continue n'est pas une exclusivité de l'administration, elle 
apparaît également prioritaire dans l'agenda de recherche au niveau des sciences de l'éducation?

La principale nouveauté, dans le domaine de la formation continue des enseignants, a été constituée par la création à partir de 1992 des centres de formation des associations d'établissements $(\mathrm{CFAE})^{8}$. Ils sont organisés selon une base territoriale: tous les établissements scolaires (de la maternelle au secondaire) qui s'inscrivent dans un même territoire se regroupent pour constituer une "association" qui donne lieu à un " centre de formation ». La gestion du centre est à la charge d'un directeur, élu par la commission pédagogique du centre, elle-même formée des représentants de tous les établissements associés. En avril 1993, on pouvait déjà constater l'existence d'un réseau, constitué par environ deux cents centres de formation, correspondant à un ensemble d'environ 120000 enseignants et couvrant l'ensemble du territoire national.

La création des CFAE s'inscrit dans une tradition qui remonte à la mise en place des premiers centres d'enseignants, en Grande-Bretagne, au début des années soixante : ils correspondaient à une tentative de trouver des modalités de formation des enseignants moins formalisées et plus interactives avec les pratiques professionnelles et les problèmes quotidiens des enseignants. Malgré les spécificités des différentes expériences nationales, les centres d'enseignants ont en commun le fait de s'adresser aux enseignants en tant qu'individus, en le définissant comme les «clients» des services rendus par les centres.

17 Au contraire, les CFAE nés, en principe, d'une démarche associative des établissements scolaires au niveau local, définissent, théoriquement, comme des clients non plus les enseignants pris individuellement, mais plutôt les organisations où ils travaillent, c'està-dire les écoles. La principale originalité des CFAE réside dans cette caractéristique qui leur ferait trouver l'essentiel de leur pertinence dans la relation établie avec l'établissement. Autrement dit, les CFAE sont nés sous le signe de la rhétorique de la "formation centrée sur l'école ». Malheureusement, cette rhétorique n'a pas réussi à se concrétiser.

18 En fait, si la création des CFAE a représenté un élargissement virtuel des marges d'initiative et des capacités d'action des enseignants et des établissements scolaires, cette virtualité a été fortement limitée à cause du paradoxe qui a marqué le processus de mise en place des CFAE : on faisait appel à un mouvement associatif local, mais... pour concrétiser une initiative dont l'État a clairement gardé tout le contrôle. En effet, le pari de l'administration centrale dans la création et dans le développement des CFAE ne devient compréhensible qu'à la lumière de deux motivations de fond: la première était celle d'appliquer la réforme et de garantir son succès, par le biais d'un accroissement de formation; la deuxième consistait à utiliser les CFAE comme des extensions administratives capables de garantir l'exécution des programmes de financement européens disponibles, et très importants, depuis 1992. L'action de l'administration a donc obéi à une visée doublement instrumentale.

Le bilan qu'on peut faire de l'activité des $\operatorname{CFAE}^{9}$ met en évidence le fait que les contraintes exercées par l'administration ont pesé lourdement, sous la forme de modalités de contrôle qui ont rendu difficile la construction d'une action autonome et diversifiée par rapport aux différents contextes. Les deux instruments fondamentaux du contrôle de l'État sont, d'une part, la maîtrise du processus de financement et, d'une façon complémentaire, l'articulation directe entre la formation et la progression dans 
la carrière à partir d'un système de crédits proportionnels aux heures de formation reçue.

La dépendance des CFAE par rapport à l'administration, créée par la combinaison des règles et modalités de financement avec l'attribution de crédits de formation, a fortement contribué à faire des CFAE des organes déconcentrés sous tutelle. Une politique orientée vers l'appui au développement organisationnel des établissements et vers le développement professionnel des enseignants supposerait, au contraire, comme une condition nécessaire une "dépendance " accrue des CFAE par rapport à leur environnement local et immédiat. Cette dépendance permettrait de les faire apparaître non plus comme un organe déconcentré de l'administration mais, plutôt, comme l'expression d'un réseau d'établissements scolaires, fondé sur une démarche associative. Cette politique supposerait, bien évidemment, des établissements scolaires plus autonomes, capables d'intégrer dans leurs préoccupations la gestion de leurs ressources humaines.

21 La convergence d'intérêts et l'action combinée de l'administration, des entités de formation et des formateurs (course aux financements), aussi bien que des enseignants (course aux crédits de formation), a favorisé, dans le cadre d'un marché de la formation, l'affirmation claire de stratégies orientées vers la «consommation » de la formation. L'offre de formation mise en place par les CFAE n'a pas réussi à dépasser une double extériorité par rapport aux enseignants et aux établissements.

L'offre de formation est marquée par l'uniformité et la standardisation, en ce qui concerne les modalités, les contenus, la durée : il s'agit presque exclusivement d'un ensemble de stages ponctuels, présentés sous la forme d'un catalogue et orientés vers la qualification individuelle de chaque enseignant. La formation dirigée vers des équipes et répondant au développement de projets d'action dans des établissements scolaires est pratiquement inexistante. Malgré la rhétorique sur la formation "centrée sur l'école ", on a gardé comme point de repère structurant la référence à un modèle de rationalité technique qui envisage la pratique professionnelle des enseignants comme une pratique individuelle, qui aurait lieu dans un vide social et qui aurait avec la théorie un simple rapport d'application. Bref, la formation continue des enseignants est restée prisonnière de la forme scolaire et ils continuent d'être formés de la même façon que les écoles traditionnelles forment les enfants ${ }^{10}$.

23 Le dilemme vécu par les CFAE, placés dans une dynamique définie par deux pôles contradictoires, celui de la tutelle et celui de l'autonomie, exprime un dilemme d'une portée plus vaste. Le débat autour de l'avenir des CFAE représente, dans une large mesure, la meilleure synthèse des contradictions et des débats qui, dans le passé récent, ont traversé le champ de la formation continue des enseignants.

\section{L'établissement scolaire et l'autonomie}

Le domaine des sciences de l'éducation a été marqué, pendant les années quatre-vingt, par le phénomène qu'on a désigné par l'expression "découverte de l'école ». Cette découverte a correspondu, dans un premier moment, à une plus grande visibilité de l'établissement scolaire en tant qu'objet social et, dans un deuxième moment, à la transformation de cet objet social en un nouvel objet d'étude. Malgré les similitudes entre les systèmes éducatifs français et portugais, cette émergence de l'établissement 
scolaire envisagé comme un niveau privilégié d'analyse et d'intervention a suivi des parcours différenciés dans les deux pays.

L'importance accordée à l'établissement scolaire remonte, en France, au début des années quatre-vingt et à la politique volontariste conduite par le ministre Alain Savary en visant la rénovation pédagogique des établissements. Le concept de projet d'établissement est devenu un élément structurant des politiques éducatives et un point de repère fondamental dans les débats sur l'autonomie et l'identité des établissements. Comme l'a bien signalé Derouet ${ }^{11}$ en France, en ce qui concerne l'émergence de l'établissement scolaire, «l'objet politique a précédé l'objet scientifique ». Au Portugal, le chemin a été, dans une certaine mesure, l'inverse. Le besoin de penser l'établissement scolaire comme une totalité (au niveau de l'action et au niveau de la production de connaissances) est associé à des dynamiques internes au champ social des pratiques éducatives, dans le cadre de projets d'intervention (situés dans la périphérie du système) dominés par le souci d'articuler, dans un seul processus, les dimensions de la recherche, de l'innovation et de la formation. La construction de dispositifs de formation des enseignants, susceptibles de faire coïncider les espaces et les temps de travail avec des espaces et des temps de formation, est à l'origine d'une réflexion systématique sur l'établissement scolaire. La formation "centrée sur l'école" a donc constitué le point de départ pour contribuer à un renversement de perspective (d'un système scolaire vers un système d'écoles) qui a rendu à l'établissement scolaire, au moins dans la théorie, le rôle d'unité-pivot de tout le système éducatif. En effet, à la fin des années quatre-vingt, l'administration s'est appropriée un ensemble de concepts - autonomie, projet d'établissement, projet éducatif local, formation centrée sur l'école - qui sont devenus des mots clés de la doctrine officielle. Malheureusement, cette appropriation est restée dans une large mesure au niveau de la rhétorique. Pourtant, il est indéniable que le problème de l'autonomie des établissements scolaires s'est affirmé comme une des préoccupations fondamentales de la politique éducative. La difficulté à traduire, par des mesures concrètes et efficaces, cette volonté politique de décentraliser découle, de notre point de vue, de trois limitations principales.

La première limitation concerne le fait d'envisager le problème de l'autonomie des établissements comme un problème de gestion qui serait dissociable d'autres problèmes : les contenus des programmes, l'évaluation des élèves, la construction de la réussite pour tous, etc. Dans cette perspective, le problème de l'autonomie devient un problème parmi d'autres, envisagé - d'une façon réductrice - comme susceptible d'être résolu par la recherche d'une formule technique plus parfaite, en ce qui concerne les modalités de gouvernement des établissements. Cette manière de voir reste tributaire d'une approche analytique et fragmentée qui ignore l'unité et la globalité systémiques de l'établissement scolaire. Elle se traduit par une multiplication et une juxtaposition stratifiée de mesures diverses, de caractère normatif, qui contribuent à compliquer le fonctionnement de l'établissement et à perturber cognitivement les acteurs. Or le problème de l'autonomie ne constitue pas un problème parmi d'autres mais, précisément, il est le nœud gordien qui matérialise une imbrication complexe de problèmes supposant une approche holistique. L'autonomie des établissements scolaires conduit à repenser leur organisation pédagogique, leur logique de fonctionnement, leurs rapports avec l'administration et avec l'environnement local (écoles voisines, autorités locales, parents, etc.). Dans cette perspective, l'autonomie 
constitue le point de départ et l'idée structurante qui peuvent guider un processus de transformation de l'école.

Une deuxième limitation correspond au fait que l'administration reste tributaire de la recherche d'une solution idéale (le one best way) qui se matérialiserait dans un normatif légal tendanciellement parfait, définissant sans ambiguité les modalités de gouvernement des établissements scolaires applicables dans tous les cas. Cette manière de voir définit le problème de l'autonomie comme un problème fermé (il y aurait une «bonne» solution) et simple (il faudrait, tout simplement, produire une nouvelle loi sur la gestion). Cette perspective s'oppose à ce que nous savons du fonctionnement réel des établissements : la complexité et la singularité des problèmes réels à résoudre se traduisent par une double diversité - celle des contextes et celle des solutions. La réalité du fonctionnement du système est plurielle et elle s'alimente de la capacité créative des acteurs locaux. La prétention à bâtir l'autonomie des établissements à partir de critères d'uniformité est, nécessairement, vouée à l'échec.

La troisième limitation est une conséquence du fait que l'administration continue à utiliser une méthodologie autoritaire, basée sur la prescription et le contrôle, même si les mesures prises visent, du moins au point de vue théorique, à produire un accroissement de l'autonomie des établissements scolaires. L'exemple des centres de formation des associations d'établissements est, à cet égard, un cas très illustratif : les établissements ont été "obligés " de s'associer, selon des découpages administratifs souvent imposés. Ensuite, ils ont été soumis à un ensemble de mécanismes bureaucratiques de contrôle qui tendent à avoir pour effet de rendre les établissements associés moins autonomes. L'usage d'une méthodologie autoritaire et centralisatrice pour imposer l'autonomie a comme conséquence de placer les établissements dans une situation de double contrainte proche des paradoxes du type «je t'ordonne de ne pas m'obéir ». La possibilité d'éviter ce cercle vicieux exige que le problème soit posé autrement.

La distinction proposée par Barroso ${ }^{12}$, entre les notions d'autonomie "décrétée » et d'autonomie «construite» est, à ce propos, très importante. Selon cet auteur, l'autonomie, qui est toujours relative, est le résultat d'un équilibre de forces au niveau d'un établissement singulier, entre différentes sources de pouvoir (internes et externes). Dans cette perspective, l'autonomie est une construction sociale qui «ne préexiste pas à l'action des individus » et donc, à la limite, l'autonomie décrétée qui est sous-jacente aux stratégies réformistes n'a pas vraiment d'existence. On peut décréter des normes pour réguler le partage des pouvoirs et l'exercice des compétences. Ces normes peuvent entraver ou, au contraire, faciliter l'autonomie de l'établissement, pourtant «elles sont incapables par elles-mêmes de créer ou de détruire cette autonomie ».

31 Dans cette perspective, la construction de l'autonomie se confond avec la production de nouvelles façons collectives d'agir, d'interagir et de penser l'action, ce qui renvoie à un processus collectif d'apprentissage. Les instances externes à l'établissement sont alors appelées à jouer un rôle de régulation nouveau: celui d'aider les établissements à apprendre à bien fonctionner, en fonction de leurs caractéristiques particulières et de la singularité de leur environnement.

32 Cette conception de la construction de l'autonomie des établissements comme un processus d'apprentissage que l'administration peut et doit favoriser est à la base d'un ensemble de propositions élaborées par J. Barroso, à la suite d'une demande du 
ministre de l'Éducation, qui établissent une orientation stratégique originale, en rupture avec les méthodes précédentes. On propose une distinction entre différents niveaux d'autonomie et une démarche contractuelle (contrats d'autonomie) qui permettraient de respecter les spécificités et les rythmes des différents établissements dans l'accès à des niveaux plus élevés d'autonomie. L'encadrement légal pour l'autonomie des établissements abandonnerait la prétention de tout prescrire, en laissant aux établissements une marge d'initiative assez large pour trouver des modalités singulières d'organisation. La concrétisation de cette orientation (articulée avec un processus plus global de décentralisation administrative) supposerait l'acceptation de la diversité réelle des établissements qui pourraient, dans une période large de transition, être soumis à des normes légales différenciées. La publication très récente de ces propositions ${ }^{13}$, représente une contribution innovatrice qui marquera le débat sur cette question dans l'avenir proche. On espère aussi que ces propositions pourront subir l'épreuve des faits.

\section{L'école et l'espace local}

Un autre thème qui domine les débats sur les politiques éducatives actuelles, au Portugal, est celui de la "territorialisation » de l'action éducative. Il est, d'ailleurs, étroitement imbriqué avec le problème de l'autonomie des établissements scolaires, c'est-à-dire qu'ils ne peuvent pas être pensés de façon séparée. L'importance de cette thématique découle de trois facteurs principaux :

- le premier concerne la reconnaissance, au Portugal comme ailleurs, de la croissante difficulté à gouverner, à partir du centre, des systèmes éducatifs qui sont devenus des machines très lourdes, en conséquence de leur expansion rapide et linéaire. Les politiques de décentralisation correspondent, donc, à un effort d'implication des instances périphériques dans la conception et l'exécution des politiques éducatives;

- le deuxième facteur correspond à une effective diffusion des fonctions d'éducation et de formation qui contribue à briser le monopole éducatif de l'école, mais aussi à remettre en cause l'idée de l'existence d'un espace national envisagé comme homogène. Au contraire d'une traditionnelle délocalisation de l'action éducative, caractéristique de l'institution scolaire, ce fait invite à une démarche d'inscription spatiale nouvelle des pratiques éducatives, soit au niveau d'un établissement ou d'un réseau d'établissements, soit d'un territoire avec une cohérence physique, administrative et sociale ;

- un troisième facteur correspond à une forte revalorisation du local en ce qui concerne la mise en œuvre de politiques intégrées de développement fondées sur les principes de l'endogénéité et de la participation. L'action éducative devient une dimension intrinsèque aux processus de développement, multidimensionnels et globalisés, au niveau d'un territoire.

Les trois facteurs énoncés tendent à converger vers la mise en valeur de la pertinence de penser en termes de politiques éducatives au niveau local qui, comme le disent Chariot et Beillerot ${ }^{14}$ doivent être "construites " et non seulement "déduites " des politiques établies au niveau national. Cette construction des politiques éducatives au niveau local se justifie pour deux raisons majeures. D'une part, il n'est pas possible d'isoler les problèmes purement éducatifs. Les dimensions sociales et éducatives apparaissent comme indissociables, ce qui fait appel à l'action convergente de plusieurs institutions et de plusieurs sortes d'acteurs sociaux. D'autre part, dans la mesure où ce 
mode de socialisation est devenu dominant dans nos sociétés, l'école est appelée à construire des réponses à des problèmes sociaux nouveaux, mais elle est dans l'impossibilité de le faire tout seule, parce que les problèmes sont territorialisés dans un double sens: certains problèmes sont concentrés dans certains territoires, les problèmes sont fortement contextualisés et, donc, réfractaires à des solutions uniformes imposées d'en haut.

Les politiques officielles dans ces domaines restent encore à un stade très embryonnaire, mais cela n'empêche pas que, à partir de l'initiative des acteurs locaux, les expériences de globalisation de l'action éducative au niveau local se multiplient. Dans cette action éducative localement globalisée la convergence d'intérêts, de ressources et d'actions a tendance à être progressivement élargie à une multiplicité de partenaires locaux tels que les parents, les entreprises, les associations culturelles, les musées, les bibliothèques publiques, les centres de santé, etc. Ceci signifie que le partenariat est devenu un mot clé pour décrire et comprendre une riche diversité d'expériences novatrices ${ }^{15}$ dont la valeur prospective pourra alimenter une stratégie inductive de la part de l'administration.

Dans ce domaine de la territorialisation de l'action éducative la donnée la plus récente et actuelle correspond au lancement, par le ministère de l'Éducation, depuis juillet 1996, d'un programme d'intervention, à niveau national, qui consiste à créer des territoires d'intervention éducative prioritaire (TEIP). Ce programme reprend, d'une façon claire, la tradition qui remonte aux expériences anglaises des années soixante et aux expériences françaises mises en place depuis 1981 (les ZEP). Le programme apparaît donc centré sur la lutte contre l'abandon, l'échec scolaire et l'exclusion, et il se fonde sur l'idée de discrimination positive dans l'attribution de ressources. Simultanément, le programme intervient dans un autre registre, celui de favoriser une action concertée entre les établissements et d'autres partenaires d'un même territoire (envisagé comme un bassin de formation).

Les caractéristiques du système éducatif portugais, que nous avons déjà évoquées d'une façon sommaire, rendent inutile la justification de l'intérêt de cette mesure de politique éducative, notamment au niveau de la scolarité obligatoire. D'autre part, sa création très récente ne conduit pas à faire des bilans qui exigeront un peu plus de temps. Il est cependant possible, et peut-être utile, d'essayer de comprendre le sens, mais aussi les contradictions que cette mesure peut soulever. La création des TEIP exprime sans doute le souci de l'administration d'utiliser des stratégies de territorialisation pour favoriser l'émergence d'une offre éducative plus pertinente et capable de favoriser la réussite des élèves. Il y a, pourtant, un certain nombre d'aspects qui posent problème et qu'on peut énoncer.

La méthodologie qui consiste à créer des regroupements d'établissements à partir d'une initiative centralisée et de critères administratifs fait ressortir une contradiction avec le souhaitable accroissement de l'autonomie et de la créativité des établissements. D'un autre côté, cette centration sur le réseau scolaire amène à sous-estimer le caractère social plus global des problèmes en présence, en accentuant sa dimension scolaire.

Cette vision "scolaire » est confirmée par le rôle mineur attribué aux mairies et aussi par la nature des organes de régulation créés : un conseil pédagogique de territoire qui reproduit et double le conseil pédagogique des établissements (collèges), avec la traditionnelle représentation disciplinaire. 
40 L'importance d'une articulation interactive avec l'environnement local, le développement d'activités d'animation, la mise à profit d'activités éducatives non formelles sont encouragés, ce qui est sans doute positif. Il reste à savoir quel type de rapport pourra s'établir entre cette orientation et une action à l'intérieur de l'école qui reprend des solutions pédagogiques fort discutables (« classes spéciales » avec des plans d'étude alternatifs). Le possible effet de stigmatisation négative des élèves et des zones choisies pour intervenir risque bien de produire des conséquences non désirées. La réussite ne peut pas être construite dans une relation de négativité.

41 Bref, la question fondamentale qui se pose est celle de savoir si cette initiative va se réduire à une intervention interne au système scolaire structurée par une stratégie d'éducation compensatoire ou si, par contre, elle va s'orienter dans le sens de la construction de politiques éducatives locales, fondées sur une démarche partenariale. Très souvent, on parle de territoires éducatifs, mais on pense, effectivement, à des territoires scolaires. L'évolution de cette expérience nous dira si ce sera le cas.

\section{L'éducation des adultes}

Le taux de personnes âgées de plus de quinze ans qui sont analphabètes est, au Portugal, le plus élevé de l'Union européenne (12\% selon les données du recensement de la population de 1991). Ces chiffres sont plus élevés dans les couches les plus âgées, parmi les femmes et dans les régions rurales de l'intérieur, où le nombre d'analphabètes peut aller jusqu'à un quart de la population, comme c'est le cas de la région de l'Alentejo ${ }^{16}$.

43 Ce phénomène devient très significativement amplifié si on envisage le problème au niveau des capacités réelles de la population pour exercer, au quotidien, les compétences de base indispensables dans une société moderne. Un rapport récent, publié sur l'initiative du conseil national de l'Éducation ${ }^{17}$, a mis en évidence l'ampleur du phénomène, commun aux pays les plus développés et scolarisés, de l'illettrisme. Cette étude a contribué à mieux comprendre les limites d'une politique d'alphabétisation centrée sur les personnes âgées qui ne sont jamais allées à l'école. La prise de conscience du phénomène a aussi contribué, d'une part, à mieux saisir les liens entre l'éducation scolaire des enfants et l'éducation des adultes et, d'autre part, à relancer le débat sur un thème, l'éducation des adultes, qui paraissait oublié.

Dans notre passé récent, on a connu deux moments forts d'investissement concernant l'éducation des adultes qui se sont traduits par des expériences riches et significatives dans ce domaine. Le premier moment remonte à la période de deux ans qui a suivi la révolution d'avril 1974. Pendant cette période-là, la politique d'éducation des adultes a été structurée par une orientation stratégique qui se proposait de valoriser, soutenir et stimuler les manifestations de culture populaire à partir d'initiatives de base ${ }^{18}$. Cette volonté d'instaurer des modalités nouvelles de relation entre les organismes officiels et les organismes populaires traduisait une politique d'articulation entre l'éducation, la construction d'une conscience civique et les processus de développement local. Cette période d'éducation populaire (très influencée par les idées de Paulo Freire) a été éphémère mais elle a laissé des marques qui seront reprises quelques années plus tard (début des années quatre-vingt) dans le cadre de la conception et de l'exécution d'un plan national d'alphabétisation et d'éducation de base des adultes (PNAEBA). 
Le PNAEBA (bien que loin de correspondre aux espoirs qu'il a suscités) a constitué, soit dans le domaine des conceptions des politiques éducatives, soit dans le domaine de la mise en œuvre expérimentale de quelques programmes, une référence très positive d'innovation. Soulignons, notamment, le rôle pionnier des expériences des « approches territoriales intégrées » et, en particulier, leurs contributions méthodologiques ${ }^{19}$ à la construction de nouvelles façons de penser et d'agir dans le champ de la formation des adultes.

Malgré ces ilôts d'innovation, il faut reconnaître que l'éducation des adultes n'a jamais correspondu, pendant les vingt dernières années, à une priorité de la politique éducative. Son caractère marginal s'est même accentué pendant les années quatrevingt et quatre-vingt-dix, au cours desquelles on a assisté à une dévalorisation, une fragmentation et une désarticulation du secteur de l'éducation des adultes. Cette évolution très négative, caractérisée par Licinio Lima $^{20}$, n'est pas dissociable, selon cet auteur, d'une vision instrumentale de l'éducation, basée sur la «rationalité » et l'« efficacité ", tributaires des conceptions néolibérales.

47 La scolarisation de l'éducation des adultes constitue l'autre trait majeur qui peut caractériser cette évolution : d'un côté, parce que ce sous-système est resté de plus en plus prisonnier dans ses conceptions et dans ses pratiques pédagogiques du "paradigme scolaire», d'autre part, parce qu'en se réduisant progressivement à la dimension de l'enseignement récurrent il est devenu un appendice du système scolaire régulier, en accueillant les jeunes qui échouent ou abandonnent de façon précoce l'école régulière ${ }^{21}$.

4 L'évolution brièvement décrite, aussi bien que les caractéristiques spécifiques de la réalité éducative portugaise, aident à comprendre pourquoi on assiste aujourd'hui à un renouveau du débat autour des politiques d'éducation des adultes. Dans le contexte portugais, leur avenir n'est pas dissociable de celui d'une éducation de base démocratique pour tous les citoyens.

\section{Redonner un sens à l'école}

Le débat sur l'école, au Portugal, est traversé par deux perspectives distinctes: la première, celle qui est dominante, cherche, à partir d'une rationalisation technique des démarches éducatives, un accroissement de l'efficacité de l'école. La deuxième perspective a comme point de départ un diagnostic de nature différente : le problème fondamental de l'école ne serait pas son efficacité mais plutôt sa légitimité et sa crédibilité sociale. Cette perspective de l'existence d'une crise de légitimité est celle qui peut fonder la pertinence d'envisager des changements en profondeur dans l'institution scolaire, en conduisant à une démarche de réinvention de l'école.

Dans ce processus de réinvention, la gestion de la diversité reste le point crucial. Historiquement, l'organisation scolaire que nous avons héritée a répondu à cette question à travers l'homogénéisation et l'uniformité des pratiques, en réduisant sa complexité interne de façon à pouvoir «enseigner à beaucoup d'élèves comme s'il s'agissait d'un seul $\aleph^{22}$. La réinvention de l'école appelle de nouvelles manières de donner des réponses pertinentes à une croissante diversité des publics scolaires. Elle appelle en premier lieu à une diversification interne de l'organisation scolaire qui puisse lui permettre d'évoluer d'un système de répétition d'informations vers un 
système de production de savoirs, en devenant un lieu où se multiplient les opportunités d'apprendre à partir d'une action interactive des élèves entre eux, avec les enseignants et avec l'environnement scolaire et local.

51 L'éclatement de l'institution scolaire en plusieurs fonctions analytiquement indépendantes donne lieu à une " école multiple » qui met au premier plan le rôle des acteurs locaux, les seuls à pouvoir donner une configuration spécifique et contextualités aux différentes logiques en présence ${ }^{23}$. Du point de vue de ces auteurs, c'est cette progressive autonomisation des différentes fonctions de l'école (intégration, distribution, subjectivation) qui fait émerger la notion de construction de l'expérience scolaire. Cette perspective aide à comprendre pourquoi les systèmes scolaires centralisés sont devenus progressivement ingouvernables et, donc, l'autonomie des établissements scolaires peut apparaître aujourd'hui, d'une certaine façon, comme une fatalité.

L'émergence et la visibilité de l'action des acteurs locaux à partir de l'utilisation du concept de construction de l'expérience scolaire conduit à mettre en premier plan, comme question clé pour l'avenir de l'école, le problème du sens du travail scolaire ${ }^{24}$ aussi bien que la nature des rapports au savoir ${ }^{25}$. Envisager la problématique de l'école du point de vue de la construction du sens conduit à ne pas dissocier les problèmes des élèves des problèmes des enseignants. Leur destin devient commun dans la mesure où cette construction ne peut qu'être une œuvre commune, dans laquelle tous les participants se transforment et apprennent.

53 Au Portugal, comme ailleurs, il y a des gens qui regardent avec nostalgie l'école d'hier. $\mathrm{Au}$ contraire, nous pensons que l'âge d'or de l'école ne se trouvera que dans un avenir où les élèves, redevenus enfants, seront des producteurs de savoirs et où ils auront, comme professeurs, des adultes disponibles et capables d'apprendre avec eux.

\section{NOTES}

1. Público, le 3 juin 1997.

2. R. Canário, « O professor e a produçéão de inovações, Colpable » Educação e Sociedade, 1993, 4, p. 97-121.

3. B. Campos (org.), Investigação e inovação para a qualidade das escolas, Lisboa, IIE, 1996.

4. A. Benavente, "Os projectos Inovar educando/Educar inovando", in: B. Campos (org.), A investigação e inovação para a qualidade das escolas, Lisboa, IIE, 1996.

5. R. Canário et alii, Mediatecas escolares. Génese e desenvolvimento de uma inovação, Lisboa, IIE, 1994.

R. Canârio, "Mediatecas escolares. 0 desenvolvimento de uma reforma no quadro de uma inovação ", Educação, Sociedade e Culturas, 2, p. 91-108, 1994.

6. M. Crozier, La crise de l'intelligence. Essai sur l'impuissance des élites à se réformer, Paris, Interéditions, 1995.

7. A. Nóvoa, «O IIE e a investigação educacional », in: B. Campos (org.), A investigação e inovação para a qualidade das escolas, Lisboa, IIE, 1996. 
8. R. Canário, «La formation continue des enseignants au Portugal : l'expérience des centres de formation, au niveau local », in : Actes du Colloque International de l'AFEC, organisé au CIEP de Sèvres du 18 au 20 mai 1995, 1995, p. 113-125.

9. J. Barroso et R. Canário, «Centras de formaçéão de associaçéões de escolas : de uma lógica de tutela a uma lógica de autonomia », Inovação (8), 3, 1995, p. 263-294.

10. D. Schon, «Formar professores como profissionais reflexivos", in: A. Nóvoa (org.), As organizações escolares em análise, Lisboa, D. Quixote/IIE, 1992.

11. J. -L. Derouet, - Une sociologie des établissements scolaires : les difficultés de construction d'un nouvel objet scientifique », in : Revue française de pédagogie, 78, 1987, p. 86-108.

12. J. Barroso, « $O$ estudo da autonomia da escola: da autonomia decretada a autonomia construída », in : J. Barroso (org.), O estudo da escola, Porto, Porto Editora, 1996.

13. J. Barroso, Autonomia e gestééão das escolas, Lisboa, ME, 1996.

14. B. Charlot et J. Beillerot, La construction des politiques d'éducation et de formation, Paris, PUF, 1995.

15. M. B. Canário, «Partenariado local e mudança educativa », Inovação (8), 1 e 2, Bettencourt, 1995, p. 151-166.

16. M.J. Esteves, Os novos contornos do analfabetismo. Analfabetismo ou iletrismo. 0 que é ? Quem são? Onde estéão ?, Lisboa, DEB, 1995.

17. A Benavente et alii, A literacia em Portugal. Resultados de uma pesquisa extensiva e monográfica, Lisboa, ICS/Universidade de Lisboa, 1996.

18. A. Melo et A. Benavente, Expériences d'éducation populaire au Portugal, 1974-1976, Paris, Unesco, 1978.

19. A. S. Silva, Educação de adultos. Educação para o desenvolvimento, Porto, ASA, 1990.

20. L. Lima, «Educação de adultos e construçào da cidadania democrética : para uma crítica do gerencialismo e da educação contabil », Inovação (9), 3, 1996, p. 283-297.

21. M. J. Esteves, « $\mathrm{O}$ retorno à escola : uma segunda oportunidade? Trajectórias sociais e escolares dos jovens e adultes que frequentam os cursos do ensino recorrente de adultes", Inovação (9), 3, 1996, p. 219-239.

A. S. Silva et L. Rothes, Estudo temático sobre educação de adultos, Porto, Faculdade de Economia do Porto, 1996.

22. J. Barroso, Os liceus. Organização pedagdógica e administração (1836-1960), Lisboa, FCG/JNICT, 1995.

23. F. Dubet et D. Martucelli, "Théories de la socialisation et définitions sociologiques de l'école ", Revue française de sociologie, XXXVII, 1996, p. 515-535.

24. Ph. Perrenoud, Métier d'élève et sens du travail scolaire, Paris, ESF, 1994.

25. B. Charlot, Du rapport au savoir. Éléments pour une théorie, Paris, Anthropos 1997.

\section{RÉSUMÉS}

Après la révolution des œillets (1974), le système éducatif portugais fait l'objet d'importantes réformes et/ou innovations. Les grands débats sont ceux de la formation permanente des enseignants, de l'autonomie des établissements scolaires en liaison avec les actions de déconcentration/décentralisation, de l'éducation des adultes. Au total, il s'agit bien de restructurer l'école pour lui donner du sens. 
INDEX

Mots-clés : établissement d'enseignement, formation continue, formation des enseignants, politique éducative, système éducatif

Index géographique : Portugal

\section{AUTEUR}

\section{RUI CANÁRIO}

Professeur à l'université de Lisbonne, Instituto das comunidades educativas, Setubal, Portugal. 